\title{
Systems for Monitoring Personal Protection Equipment
}

\author{
Liqun Wang, Chuansheng Wu, Qiang Fu \\ University of Science and Technology Liaoning,China \\ gykwcs@163.com
}

Keywords: monitoring personal, protection personal; equipment, promoting; worker safety

\begin{abstract}
A system for promoting the safety of workers comprises a digital imaging device positioned to capture one or more images of a predetermined viewing area. Further, the system comprises an image processor operatively associated with the digital imaging device. The image processor is configured to determine whether a person is within the predetermined viewing area of the digital imaging device. The image processor is further configured to determine whether the person is not wearing required personal protection equipment. Additionally, the image processor is configured to generate a message or control signal in response to determining the person is within the predetermined viewing area of the digital imaging device and determining the person is not wearing the required personal protection equipment.
\end{abstract}

\section{Introduction}

Workplace safety is a concern to all those present in a hazardous environment. The National Institute for Occupational Safety and Health (NIOSH) and State-based investigation provide reports on fatal occupational injuries. Based on these reports, in 2010, there were an estimated 139,064,000 civilian workers in the U.S. private and public sector employed labor force. According to the Bureau of Labor Statistics, each day, many of these workers suffer injury, disability and/or death from workplace incidents. In 2010, more than 4,500 U.S. workers died from occupational injuries. Although difficult to enumerate, about 49,000 deaths annually are attributed to work-related illnesses. In 2010, an estimated 3.9 million workers in private industry and state and local government had a nonfatal occupational injury or illness. Of those workers, 2 million were transferred, placed on work restrictions, or took time away from work. In the same year an estimated 2.6 million workers were treated in emergency departments for occupational injuries and illnesses, and approximately ,000 of these workers were hospitalized[1,2].

\section{A system for promoting the safety of persons comprising}

A system for promoting the safety of persons comprising:

a digital imaging device positioned to capture one or more images of a predetermined viewing area; and an image processor operatively associated with the digital imaging device and configured to: determine whether a person is within the predetermined viewing area of the digital imaging device; determine whether the person is not wearing required personal protection equipment; and generate a message in response to determining the person is within the predetermined viewing area of the digital imaging device and determining the person is not wearing the required personal protection equipment. wherein the image processor is further configured to determine if the person is not wearing required personal protection equipment based on one or more colors. wherein the image processor is further configured to determine if required personal protection equipment comprises of one or more symbols[3].

A method for promoting the safety of persons, the method comprising: positioning a digital imaging device to capture one or more images of a predetermined viewing area; associating an image processor operatively with the digital imaging device; determining whether a person is within the predetermined viewing area of the digital imaging device; determining whether the person is not wearing required personal protection equipment; and generating a message in response to 
determining the person is within the predetermined viewing area of the digital imaging device and determining the person is not wearing the required personal protection equipment. further comprising determining if the person is not wearing required personal protection equipment based on one or more colors. wherein the image processor is further configured to determine if required personal protection equipment comprises of one or more symbols[4,5].

The presently disclosed invention is now described in more detail. FIG. 1 is an illustration showing an example system for identifying an individual wearing personal protective equipment (PPE) that includes identifying symbols and/or colors located on the PPE within view of a digital imaging device according to embodiments of the present invention. The PPE may be comprised of gloves , a vest , boots , a hard hat , a pair of goggles , or a mask , as an example. The individual may include a worker, supervisor or visitor in a residential, commercial or industrial environment, and may not include those individuals not requiring PPE at that location, as a non-limiting example. For example, emergency responders may not be required to wear PPE or they may have the necessary PPE already. The identifying symbols may be of any shape or size viewable by the digital imaging device and recognizable by an image processor . A prominently displayed character or symbol(s) may be provided on a least one or more prominent portions of the individual piece of PPE where it can easily be detected by the digital imaging device .

\section{A worker wearing personal protective equipment}

With continued reference to FIG. 1, the system may also include an image processor . The image processor is operationally coupled to the digital imaging device. The image processor may be configured to receive both still and full-motion images from the digital imaging device . The digital imaging device may also include a wide angle fish eye lens. The wide angle fish eye lens may be interchanged with other types of lens, including but not limited to a zoom lens, a polarized lens, etc. Digital imaging may be also accomplished via multi lens array, stereo pair, infrared time of flight scanner/imager or structured light enabled 2D or 3D imaging, as an example. As an alternative embodiment, the individuals associated with the presence of the symbols or within the viewing area may be identified based on facial recognition or identifiable aspects of the individual. As an example, the individual may be identified based on facial characteristics, height, iris characteristics, fingerprints or other appropriate biometric parameters.

The system may also comprise hardware, software, firmware, or combinations thereof for implementing the functionality described herein. For example, the system may include at least one processor and memory to enable the image processor to compete more complex tasks. For example, the processor may be wirelessly coupled to a database (not shown) for data logging the results of each image analysis. In this manner, reporting the individual, date, time and items worn, as a non-limiting example. Subsequent to the image analysis described above, the system may generate a message or control signal and transmit the message to an annunciator or a machinery control processor . As an example, the message may be the control signal transmitted to the machinery control processor .

\section{Method of operation of the digital imaging device and image processor}

Comprising the digital imaging device and the image processor for detecting the presence of the individual, determine if all required PPE are present and generating a message based on the determination may be provided according to embodiments of the present invention. The optional wide angle optical lens may be affixed to the digital imaging device . The digital imaging device may be positioned in a work area, which may be a job site, factory floor, or commercial/industrial area where one or more pieces of personal safety equipment is required. The individual or worker may be viewed from the waist up, head up and/or feet alone within a predetermined viewing area of the digital imaging device. The predetermined viewing area may be an area sufficient for proper viewing of the individual and any worn PPE with sufficient resolution to determine the type of indicia placed on the PPE , whether shape or color based. 


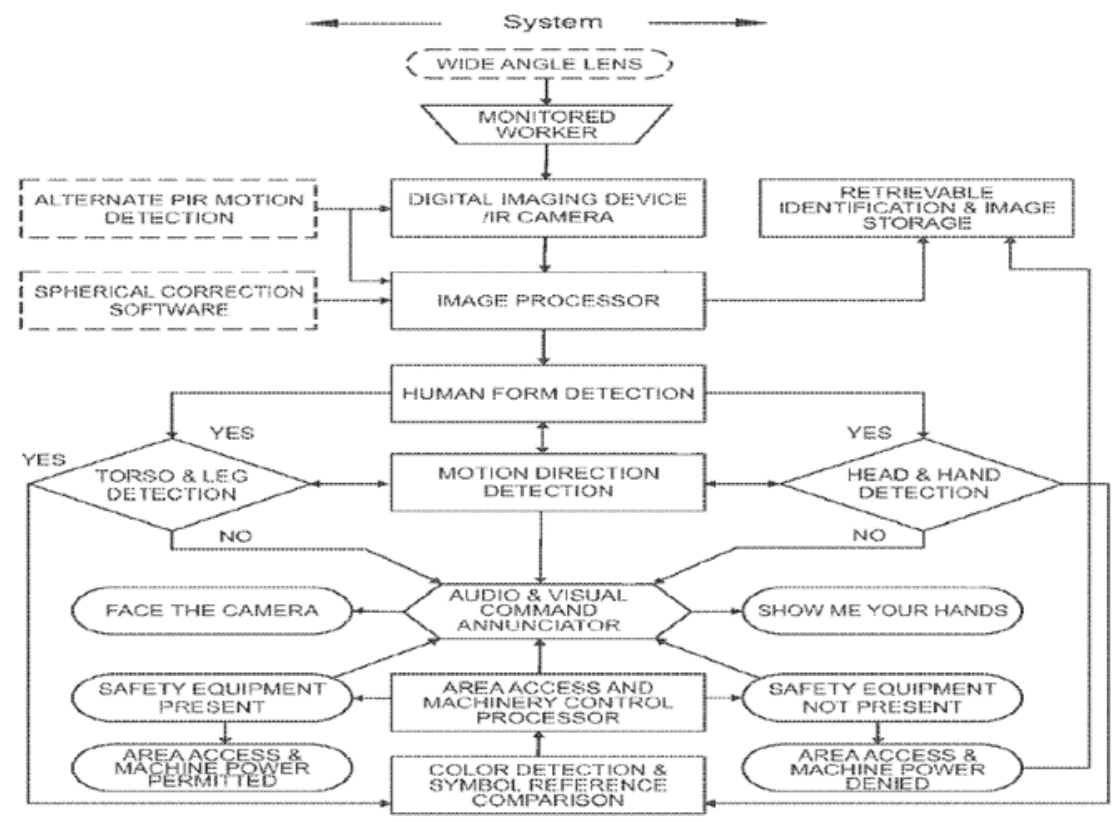

FIG. 1 is a flowchart showing an example

With continued reference to FIG. 1, different resolutions of the image may be analyzed to assist in object recognition. Initial resolution may be defined by detection of any large, presumably human object detection changing the background scene as determined by the motion direction detection routine . A system for detecting directional motion is disclosed in U.S. Pat. No. 6,707,486 to Millet et al., the disclosure of which is incorporated by reference in its entirety herein. Human form detection and background subtraction by the human object detection further refines the detection scheme as coarse localization focuses in on the human form within the captured image to reduce the number of pixels and thereby reduce the computational complexity. in order to facilitate the removal of the ambient background from the image. To reduce illumination effects on the image, before classification, each sub-image may be normalized with respect to brightness and contrast. This sub-image minus the ambient background preferably has a fixed sized so as to reference against a library of know shapes and forms. Since the segmentation process itself may be inherently ambiguous, it may be preferably coupled to the symbols shape for identification.

\section{Conclusion}

The flowchart and block diagrams in the Figures illustrate the architecture, functionality, and operation of possible implementations of systems, methods, and computer program products according to various embodiments. In this regard, each block in the flowchart or block diagrams may represent a module, segment, or portion of instructions, which comprises one or more executable instructions for implementing the specified logical function(s). In some alternative implementations, the functions noted in the block may occur out of the order noted in the figures.

\section{References}

[1] CLOUD COMPUTING IN APPLICATION OF PERSONALIZED LIBRARY SERVICES[A]. Proceedings of 2012 International Symposium on Computer Science and Technology(ISCST'2012)[C]. 2012

[2] Studies on Application of Cloud Computing Techniques in GIS[A]. Proceedings of 2013 Second IITA International Conference on Geoscience and Remote Sensing (IITA-GRS 2013) Volume 1[C]. 2013

[3] Cloud Computing-based Product Collaborative Design and Simulation[A]. 2013 ETP/IITA Conference on System Science and Simulation in Engineering(SSSE 2013) Proceedings[C]. 2013 
[4] The Impacts of Cloud Computing on Geospatial Information Science[A]. Proceedings of 2013 2nd International Conference on Multimedia and Computational Intelligence (ICMCI 2013)[C]. 2013

[5] CLOUD COMPUTING:NEW OPPORTUNITY FOR INFORMATIONIZATION OF UNIVERSITY EDUCATION[A]. Proceedings of 2012 International Symposium on Computer Science and Technology (ISCST’2012) [C]. 2012 